\title{
Epidemiology of cervical spondylotic myelopathy and its risk of causing spinal cord injury: a national cohort study
}

\author{
*Jau-Ching Wu, M.D., Ph.D., ${ }^{1-3}$ Chin-Chu Ko, M.D., ${ }^{1-3}$ Yu-Shu Yen, M.D., ${ }^{1,2}$ \\ Wen-Cheng Huang, M.D., Ph.D., ${ }^{1,2}$ Yu-Chun Chen, M.D., Ph.D., ${ }^{2,4,5}$ \\ Laura Liu, M.D., Ph.D., ${ }^{6,7}$ Tsung-Hsi Tu, M.D., ${ }^{1,2}$ Su-Shun Lo, M.D., ${ }^{2}$ \\ and Henrich Cheng, M.D., Ph.D. ${ }^{1-3}$
}

${ }^{1}$ Department of Neurosurgery, Neurological Institute, Taipei Veterans General Hospital, Taipei; ${ }^{2}$ School of Medicine, ${ }^{3}$ Institute of Pharmacology, and ${ }^{5}$ Institute of Hospital and Health Care Administration, National Yang-Ming University, Taipei; ${ }^{4}$ Department of Medical Research and Education, National Yang-Ming University Hospital, I-Lan; ${ }^{6}$ Department of Ophthalmology, Chang Gung Memorial Hospital, Taoyuan; and ${ }^{7}$ College of Medicine, Chang Gung University, Taoyuan, Taiwan

Object. This study aimed to determine the age- and sex-specific incidence of cervical spondylotic myelopathy (CSM) and its associated risk of causing subsequent spinal cord injury (SCI).

Methods. Using the National Health Insurance Research Database (NHIRD), a 12-year nationwide database in Taiwan, this retrospective cohort study analyzed the incidence of hospitalization caused by CSM. All patients diagnosed with and admitted for CSM were identified during the study period. The CSM patients were divided into 2 groups, a control group and an operated group. An incidence density method was used to estimate age- and sexspecific incidence rates of CSM. The Kaplan-Meier method and Cox regression analyses were performed to compare the risk of SCI between the 2 groups.

Results. From 1998 to 2009, covering 349.5 million person-years, 14,140 patients were hospitalized for CSM. The overall incidence of CSM-related hospitalization was 4.04 per 100,000 person-years. Specifically, males and older persons had a higher incidence rate of CSM. During the follow-up of these patients for 13,461 person-years, a total of 166 patients were diagnosed with SCI. The incidence of SCI was higher in the control group than the operated group (13.9 vs 9.4 per 1000 person-years, respectively). During the follow-up, SCI was more likely to occur in CSM patients who were treated conservatively (crude HR 1.48, p $=0.023$; adjusted HR 1.57, p = 0.011) than in those who underwent surgery for CSM.

Conclusions. In a national cohort of eastern Asia, the incidence of CSM-caused hospitalization was 4.04 per 100,000 person-years, with higher incidences observed in older and male patients. Subsequent SCI was more likely to develop in patients who received nonoperative management than in those who underwent surgery. Therefore, patients with CSM managed without surgery should be cautioned about SCI. However, further investigations are still required to clarify the risks and complications associated with surgery for CSM.

(http://thejns.org/doi/abs/10.3171/2013.4.FOCUS13122)

$\begin{array}{lccccc}\text { KEY WORDS } & \text { cervical spondylotic myelopathy } & \bullet & \text { incidence } & \bullet & \text { spinal disorders } \\ \text { spinal cord injury } & \bullet \quad \text { national health insurance } & & \end{array}$

$\mathrm{C}$ ERVICAL spondylotic myelopathy is the most common cause of spinal cord dysfunction in modern society worldwide. Spondylosis, the cascade of degeneration in the vertebral column, encompasses diseases of a number of related structures. For example, common etiologies of CSM are formation of osteophytes, disc herniation, degenerative disc disease, and OPLL, causing spinal cord compression or ischemia. The presentation of CSM also varies greatly, ranging from quiescent or insidious symptoms to stepwise or rapidly deteriorating

\footnotetext{
Abbreviations used in this paper: $\mathrm{CSM}=$ cervical spondylotic myelopathy; NHRI = National Health Research Institutes; NHIRD = National Health Insurance Research Database; OPLL = ossification of posterior longitudinal ligament; $\mathrm{SCI}=$ spinal cord injury.

* Drs. Ko and Yen contributed equally to this work.
}

neurological function, and CSM can eventually cause quadriparesis. ${ }^{1,69,18}$ Because the natural history, optimal management strategies, and the timing of intervention and prognostic factors of CSM are unclear, CSM remains an issue of debate in neurosurgery. ${ }^{10}$

The epidemiology of CSM, including its incidence and prevalence, is seldom reported in the literature. A study from the United Kingdom reported a total of 41 patients who presented with CSM. The mean age of these patients was 68.7 years, and the study concluded that CSM predominantly affects men in their 7th decade of life. ${ }^{13}$ A Japanese study noted that the annual rate of operations per 100,000 residents in a northeastern prefecture was 5.7 and that most of these patients were in their 6th or 7 th decade of life.? 
It is reasonable to infer that patients with CSM are at a higher risk of subsequent SCI because of the predisposing factor of cervical spinal stenosis. However, evidence has been scarce for this intuitive assumption. The Joint Guidelines Committee of the American Association of Neurological Surgeons and the Congress of Neurological Surgeons suggested that among patients with cervical stenosis without myelopathy, the presence of abnormal electromyography findings or the presence of clinical radiculopathy is associated with the development of symptomatic CSM. ${ }^{10}$ Therefore, surgical decompression is warranted in these selected CSM patients. Nevertheless, the risk of SCI in patients with CSM still requires validation.

This study aimed to provide a population-based epidemiological investigation of CSM and analysis of the subsequent risk of SCI if the CSM is managed nonoperatively. The authors used the NHIRD, which is a national database provided by the NHRI of Taiwan containing 23 million administered insurants recorded from January 1997 to December 2009. This unique government-operated insurance system has an extremely high coverage of more than $99 \%$ of the population and finances health care for the entire population by offering unrestricted access to any health care provider of the patient's choice. Because of the universal coverage that this database provides, this investigation can yield a sound epidemiological overview of the incidence of CSM, patients' use of medical care, and risk of subsequent adverse events including SCI.

\section{Methods}

\section{Database}

This study used the NHIRD, which included all claims data from Taiwan's National Health Insurance program. The study was approved by the institutional review board of the Taipei Veterans General Hospital, Taiwan, because the NHIRD contains anonymous secondary data released to the public for research purposes. ${ }^{2}$

\section{Hospitalization and Surgical Procedures}

In the NHIRD, diagnosis of every admission is recorded by the ninth version of the ICD-9. During the study period, all hospitalized patients discharged with the diagnostic code for CSM (721.1) were identified from the cohort.

The incidences of hospitalization for CSM were determined by identifying individuals who were followed up for more than 1 year and newly hospitalized with the above-mentioned CSM discharge code between January 1, 1998, and December 31, 2009. The incidence rates in this study were estimated by the incidence density.

The date of the recorded incidence of CSM (the index date) was designated as the first date of each patient's specific hospitalization. Subsequent spinal surgery was determined by any event of hospitalization with procedure codes of spinal fusion (ICD-9 procedure codes 81.02-3 and 84.61-2) or spinal decompression $(03.02,03.4 \mathrm{x}$, 80.99 , and 80.51 ) within the 12 months immediately following the index date. These procedure codes included cervical laminectomy, laminoplasty, discectomy, corpectomy, and anterior or posterior spinal arthrodesis. In theory, common surgical procedures for CSM (for example, anterior, posterior, and combined anterior and posterior approaches) were all included in this analysis.

\section{Operated Versus Control Groups}

All of the CSM patients identified were divided into 2 groups. The operated group comprised individuals who underwent any of the aforementioned surgical procedures after the index date. The control group comprised subjects who did not receive the above-mentioned surgical procedures. Prior admission for cervical SCI was determined by hospital records with the corresponding discharge diagnostic codes (ICD-9 codes 952.0x and 806.0-1) before the index date. After exclusion of all prior cervical SCIs, all subjects with CSM were followed up for subsequent events of SCI.

\section{Covariates and Outcomes}

Comorbidities of CSM were identified by the presence of either diagnostic codes of outpatient records or discharge codes of hospitalization records 6 months before the index date to the date of outcome event or the end of follow-up. These comorbidities included osteoarthrosis (ICD-9 code 715.x), osteoporosis (733.0x), rheumatoid arthritis (714.x), diabetes mellitus (250.x), and hypertension (401-5.x). To further adjust baseline differences between the 2 groups, propensity scoring took into account all of these comorbidities as covariates, and propensity scores were calculated by logistic regression analysis. ${ }^{16}$

Cervical SCI was considered as the outcome of CSM and the endpoint in the present study. Hospitalization for cervical SCI was determined by using the date on the hospitalization records that had a discharge diagnostic code for cervical SCI (ICD-9 codes 952.0x and 806.0-1) after the index date.

\section{Statistical Analysis}

All data were analyzed using the STATA software for generating descriptive statistics and creating contingency tables. The incidence density method was used to estimate the age- and sex-specific incidence for CSM. The Kaplan-Meier method and log-rank tests were used to estimate and compare the incidence rates of hospitalizations for SCI. The Cox proportional hazard model with propensity scores was used to compare the incidence rates of subsequent SCI between the 2 groups after adjustment for the aforementioned covariates. A $p$ value $<0.05$ was considered statistically significant.

\section{Results}

\section{Incidence of CSM}

Between 1998 and 2009, 14,140 patients recorded in the NHIRD were hospitalized for CSM, with a total follow-up of 349.5 million person-years. The overall incidence of CSM-caused admissions was 4.04 (95\% CI 3.98-4.11) per 100,000 person-years. Age-specific and 


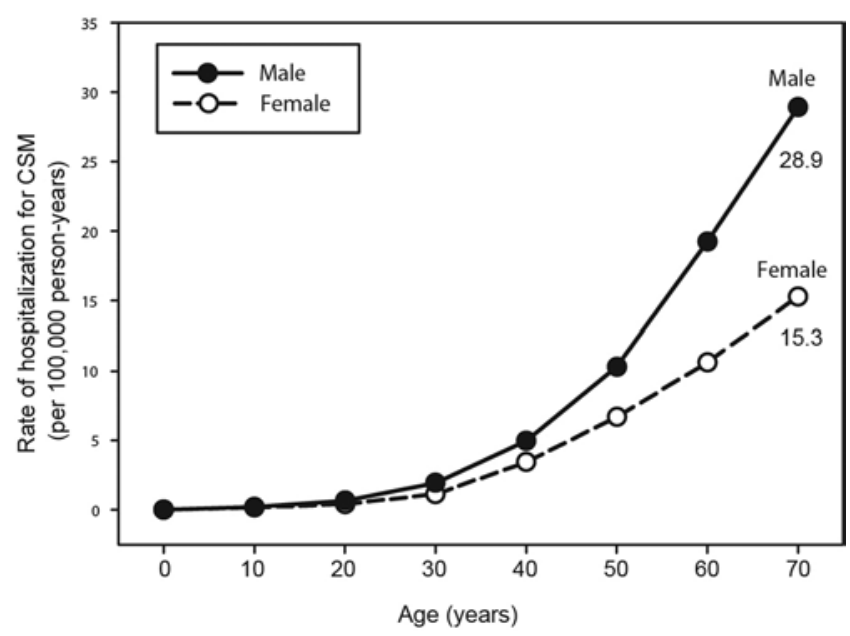

FIG. 1. Age- and sex-specific incidence rates of CSM requiring hospitalization (1998-2009).

sex-specific incidence rates are shown in Fig. 1, indicating a higher incidence of CSM in males and in older patients. The highest incidence of CSM among males and females was 28.9 and 15.3 per 100,000 person-years in patients 70 years and older, respectively.

\section{Incidence of SCI in CSM Patients}

A total of 14,140 patients with CSM were followed up for 13,461 person-years. Of these patients, 166 were subsequently hospitalized for SCI. The overall incidence of SCI in the CSM patients was 12.33 per 1000 personyears.

Patients in the control group and those in whom CSM had not been surgically managed were more likely to be hospitalized for SCI than patients who underwent surgery for CSM. The incidence rate of SCI requiring hospitalization in the control group (13.90 per 1000 person-years) was 1.48 times higher than that in the operated group (9.39 per 1000 person-years). The crude HR for hospitalized SCI was 1.48; after adjusting for comorbidities, the HR was 1.57, suggesting a more than 1.5-fold higher SCI incidence rate in the control group than in the operated group (Table 1).
The CSM patients in the operated group had a significantly lower probability of hospitalization for SCI in the 12 months of follow-up after the index date. The cumulative incidence rate of hospitalization for SCI in the control group was $1.39 \%$ (95\% CI 1.16-1.66), which was significantly $(\mathrm{p}=0.03, \log$-rank test) higher than that in the operated group $(0.94 \%, 95 \%$ CI $0.70-1.26)$ (Fig. 2). The cumulative incidence rates of hospitalizations due to $\mathrm{SCI}$ in the control and operated groups showed a parallel increase within 12 months from the index date.

\section{Discussion}

With 14,140 patients included, this study is the largest to date that has analyzed the incidence of CSM and its association with risk of SCI. This study of a comprehensive nationwide cohort surveyed over a total period of more than 12 years has shown that CSM caused hospitalization at a rate of 4.04 per 100,000 person-years. Higher incidences for CSM were found in the elderly and in male patients. The incidence of SCI in these patients with CSM was as high as 12.33 per 1000 person-years. Moreover, cervical SCI was approximately 1.5 times more likely to ensue in CSM patients who were managed without surgery than in those who underwent surgery to treat CSM. Therefore, patients with conservatively treated CSM should be cautioned about the possibility of subsequent SCI. This report is the first focused on the epidemiology of CSM on a national scale. As previously reported, CSM is more commonly seen in older and male patients. ${ }^{13}$ Furthermore, previous reports have also noted higher incidences of cervical OPLL - a known cause of CSM-among elderly and male patients. ${ }^{21}$ These results imply a degenerative nature of CSM and cervical OPLL.

One may intuitively infer that patients with CSM are at higher risk of SCI because of preexisting spinal stenosis. Although a significantly smaller spinal canal diameter has been shown in cases of CSM, ${ }^{4,12}$ evidence is scarce in the literature for supporting the theory that spinal stenosis predisposes individuals to SCI. Coexisting OPLL has been reported to be associated at a rate of $6.5 \%-10.1 \%$ in cervical SCI in different series. ${ }^{5,14,20} \mathrm{Mi}$ nor trauma or a relatively small external force can cause

TABLE 1: Incidence and hazard ratios for cervical SCI in the patients with CSM grouped by treatment method*

\begin{tabular}{lccc}
\hline & \multicolumn{3}{c}{ Value $(95 \% \mathrm{Cl})$} \\
\cline { 2 - 4 } \multicolumn{1}{c}{ Variable } & Total Sample & Operated Group & Control Group \\
\hline incidence of hospitalization for SCl† & $12.33(10.59-14.36)$ & $9.39(6.99-12.62)$ & $13.90(11.64-16.60)$ \\
no. of hospitalizations for SCI† & 166 & 44 & 122 \\
observed person-years & $13,461.44$ & $4,684.71$ & $8,776.73$ \\
crude HR & $\mathrm{NA}$ & reference & $1.48(1.04-2.14) \ddagger$ \\
adjusted HR§ & $\mathrm{NA}$ & reference & $1.57(1.11-2.22)^{\star *}$ \\
\hline
\end{tabular}

* NA = not applicable.

$\dagger$ Per 1000 person-years.

$\ddagger$ The crude hazard ratio was statistically significant $(p=0.023)$.

$\S$ The hazard ratio was adjusted for patients' age and sex, and the propensity scores for this adjustment were calculated by taking into account diabetes, hypertension, osteoporosis, rheumatoid arthritis, and osteoarthritis as covariates.

** The adjusted hazard ratio was statistically significant $(p=0.011)$. 


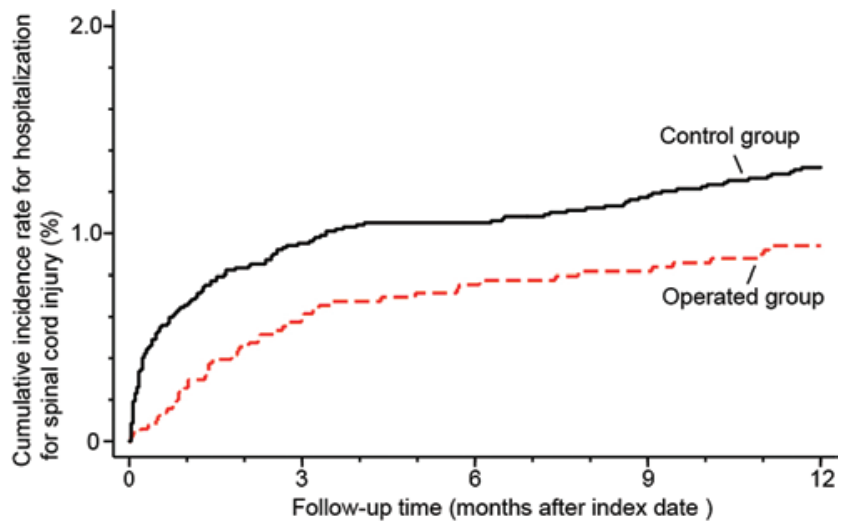

FIG. 2. Cumulative incidence rates for cervical SCI causing hospitalization in patients with CSM $(n=14,140)$.

SCI in OPLL patients because of the narrowed spinal canal in these patients. ${ }^{5,21}$ Cervical OPLL is one of the etiologies of CSM, and these 2 pathologies should therefore share many similar features. However, clinical trials have not supported the assumption that patients with spinal stenosis are prone to SCI.

The incidence rate of SCI in this study was as high as 12.33 per 1000 person-years. This rate is extremely high when compared with the incidence rate of cervical SCI for the general population (0.13 per 1000 person-years). ${ }^{19}$ The present study also demonstrated that conservatively managed CSM patients were at higher risk of SCI. Hence, CSM might be regarded as a risk factor of SCI.

The natural history of CSM is still unclear; thus, the best management and timing of any intervention remain debatable..$^{8,10,17,22}$ It has been suggested that SCI might be a part of the natural course of CSM. ${ }^{15}$ However, presentations of patients with CSM are widely variable: some CSMs are quiescent and insidious, and rapid neurological deterioration can develop after a latent period. Treatment of mild CSM may involve surgical decompression or nonoperative therapy for the first 3 years after diagnosis. ${ }^{11}$ Management of CSM is justified not only to facilitate neurological recovery but also to prevent functional deterioration or SCI. Moreover, the present study included only CSM patients who had been hospitalized. Recorded outpatients with CSM of variable severity were not included in this study. As was observed in this hospitalbased dataset, the majority of CSM outpatients might not progress to SCI. This caveat must be noted before interpreting the results in this report as being representative of the history of CSM.

There are limitations to this study. A remarkable heterogeneity existed in the group of CSM patients included here, which might have caused bias in the outcome analysis of SCI. This is an inherent limitation because the NHIRD does not incorporate records of neurological examinations. Using this database, it is also impossible to trace medical charts for evaluation of each individual's neurological function. The presence or severity of myelopathy upon hospitalization for CSM or SCI is not available for analysis and thus is subject to substantial variability. Nevertheless, all CSM patients included in the present study had been hospitalized. Admission for evaluation or treatment indicated moderately or severely symptomatic CSM, which increases the clinical relevance of this analysis.

Furthermore, selection bias might have occurred in both the control group and the operated group. Patients who were managed surgically were likely different from those who were managed only with medication and rehabilitation. This issue was addressed by propensity scoring and logistic regression analysis including all known covariates.

In addition, the coding in the NHIRD is used for billing and social welfare benefits, so it is prudently and internally monitored. Fraudulent coding is subject to heavy penalties, and a validation study has suggested the reliability of the diagnostic codes in the NHIRD. ${ }^{3}$ Because of the complete coverage of the National Health Insurance in Taiwan, the longitudinal follow-ups for subsequent SCI events are comprehensive and highly accurate. Loss of follow-up for SCI can only occur when the patient dies at the scene or moves to a foreign country. Therefore, this report provides sound and accurate epidemiological data.

The results of the present study are valuable for research into the epidemiology and natural history of outcomes among patients with CSM. Also, the data may be of help in the management of CSM, especially for the prevention of SCI. However, this study did not take into account the risks and complications associated with surgery among the patients with CSM. Whether the treatmentrelated benefit (that is, protection against SCI) can offset the risks and complications of surgery remains unclear. Future studies on a larger scale, with longer follow-up, and with a more detailed classification of the patients' neurological functions are needed to establish a treatment algorithm.

\section{Conclusions}

In a national cohort of eastern Asia, the incidence of CSM-caused hospitalizations was 4.04 per 100,000 person-years, with a higher incidence of CSM observed in older and in male patients. Subsequent SCI was more likely to develop in CSM patients who received nonoperative management than in those who underwent surgery. Therefore, patients with CSM should be cautioned about the risk of SCI if managed without surgery. However, further investigation is required to clarify the risks and complications associated with surgery.

\section{Disclosure}

The authors report no conflict of interest concerning the materials or methods used in this study or the findings specified in this paper.

This study was based partly on data from the NHRI database provided by the Bureau of National Health Insurance, Department of Health and managed by NHRI in Taiwan. The interpretation and conclusions contained herein do not represent those of the Bureau of National Health Insurance, the Department of Health, or the NHRI.

Author contributions to the study and manuscript preparation include the following. Conception and design: Chen, $\mathrm{Wu}, \mathrm{Ko}$, Huang, Liu, Tu. Acquisition of data: Chen. Analysis and interpretation of data: Chen, Wu, Ko, Huang, Tu. Drafting the article: Chen, $\mathrm{Wu}$, Liu. Critically revising the article: Chen, Wu, Yen. Reviewed 


\section{Epidemiology of CSM and the risk of SCI}

submitted version of manuscript: Chen, Wu, Yen. Approved the final version of the manuscript on behalf of all authors: Chen. Statistical analysis: Chen. Administrative/technical/material support: Lo, Cheng. Study supervision: Lo, Cheng.

\section{References}

1. Baron EM, Young WF: Cervical spondylotic myelopathy: a brief review of its pathophysiology, clinical course, and diagnosis. Neurosurgery 60 (1 Suppl 1):S35-S41, 2007

2. Chen YC, Yeh HY, Wu JC, Haschler I, Chen TJ, Wetter T: Taiwan's national health insurance research database: administrative health care database as study object in bibliometrics. Scientometrics 86:365-380, 2011

3. Cheng CL, Kao YH, Lin SJ, Lee CH, Lai ML: Validation of the national health insurance research database with ischemic stroke cases in Taiwan. Pharmacoepidemiol Drug Saf 20:236-252, 2011

4. Dong F, Shen C, Jiang S, Zhang R, Song P, Yu Y, et al: Measurement of volume-occupying rate of cervical spinal canal and its role in cervical spondylotic myelopathy. Eur Spine J [epub ahead of print], 2013

5. Endo S, Shimamura T, Nakae H, Takakuwa T, Yamada Y, Kasai T, et al: Cervical spinal cord injury associated with ossification of the posterior longitudinal ligament. Arch Orthop Trauma Surg 113:218-221, 1994

6. Fehlings MG, Skaf G: A review of the pathophysiology of cervical spondylotic myelopathy with insights for potential novel mechanisms drawn from traumatic spinal cord injury. Spine (Phila Pa 1976) 23:2730-2737, 1998

7. Kokubun S, Sato T, Ishii Y, Tanaka Y: Cervical myelopathy in the Japanese. Clin Orthop Relat Res (323):129-138, 1996

8. LaRocca H: Cervical spondylotic myelopathy: natural history. Spine (Phila Pa 1976) 13:854-855, 1988

9. Lebl DR, Hughes A, Cammisa FP Jr, O'Leary PF: Cervical spondylotic myelopathy: pathophysiology, clinical presentation, and treatment. HSS J 7:170-178, 2011

10. Matz PG, Anderson PA, Holly LT, Groff MW, Heary RF, Kaiser MG, et al: The natural history of cervical spondylotic myelopathy. J Neurosurg Spine 11:104-111, 2009

11. Matz PG, Holly LT, Mummaneni PV, Anderson PA, Groff MW, Heary RF, et al: Anterior cervical surgery for the treatment of cervical degenerative myelopathy. J Neurosurg Spine 11:170-173, 2009

12. Morishita Y, Naito M, Hymanson H, Miyazaki M, Wu G, Wang JC: The relationship between the cervical spinal canal diameter and the pathological changes in the cervical spine. Eur Spine J 18:877-883, 2009
13. Northover JR, Wild JB, Braybrooke J, Blanco J: The epidemiology of cervical spondylotic myelopathy. Skeletal Radiol 41:1543-1546, 2012

14. Okada S, Maeda T, Ohkawa Y, Harimaya K, Saiwai H, Kumamaru H, et al: Does ossification of the posterior longitudinal ligament affect the neurological outcome after traumatic cervical cord injury? Spine (Phila Pa 1976) 34:1148-1152, 2009

15. Oshima Y, Seichi A, Takeshita K, Chikuda H, Ono T, Baba S, et al: Natural course and prognostic factors in patients with mild cervical spondylotic myelopathy with increased signal intensity on T2-weighted magnetic resonance imaging. Spine (Phila Pa 1976) 37:1909-1913, 2012

16. Rubin DB: Estimating causal effects from large data sets using propensity scores. Ann Intern Med 127:757-763, 1997

17. Sadasivan KK, Reddy RP, Albright JA: The natural history of cervical spondylotic myelopathy. Yale J Biol Med 66:235242,1993

18. Tetreault LA, Karpova A,Fehlings MG: Predictors of outcome in patients with degenerative cervical spondylotic myelopathy undergoing surgical treatment: results of a systematic review. Eur Spine J [epub ahead of print], 2013

19. Wu JC, Chen YC, Liu L, Chen TJ, Huang WC, Cheng H, et al: Effects of age, gender, and socio-economic status on the incidence of spinal cord injury: an assessment using the elevenyear comprehensive nationwide database of Taiwan. J Neurotrauma 29:889-897, 2012

20. Wu JC, Chen YC, Liu L, Huang WC, Chen TJ, Lo SS, et al: Conservatively treated ossification of the posterior longitudinal ligament increases the risk of spinal cord injury: a nationwide cohort study. J Neurotrauma 29:462-468, 2012

21. Wu JC, Liu L, Chen YC, Huang WC, Chen TJ, Cheng H: Ossification of the posterior longitudinal ligament in the cervical spine: an 11-year comprehensive national epidemiology study. Neurosurg Focus 30(3):E5, 2011

22. Yarbrough CK, Murphy RK, Ray WZ, Stewart TJ: The natural history and clinical presentation of cervical spondylotic myelopathy. Adv Orthop 2012:480643, 2012

Manuscript submitted March 15, 2013.

Accepted April 19, 2013.

Please include this information when citing this paper: DOI: 10.3171/2013.4.FOCUS13122.

Address correspondence to: Yu-Chun Chen, M.D., Ph.D., Department of Medical Research and Education, National Yang-Ming University Hospital, No. 152, Xin Min Rd., 26042 I-Lan, Taiwan, ROC.email: yuchn.chen@gmail.com. 